\title{
NEW SOLUTION METHOD OF TIME- DEPENDENT SCHRÖDINGER EQUATION USING IMPROVED BOUNDARY CONDITIONS
}

\author{
C. P. SONG, M. R. LEE and K. F. YARN* \\ Far East College, Department of Electrical Engineering, \\ Optoelectronic Semiconductor Center, Hsin-Shih, Tainan, \\ Taiwan 744, Republic of China
}

(Received 30 June 2000; In final form 12 September 2000)

\begin{abstract}
Numerical solution of the time-dependent Schrödinger equation for open system structures has been impeded by the difficulty in handing open-system boundary conditions. This paper presents a new numerical method for time-dependent Schrödinger equation and a boundary condition method to simulate the interaction with ideal particle reservoirs at the structure boundaries.
\end{abstract}

Keywords: Schrödinger equation; Boundary condition

\section{INTRODUCTION}

The time-dependent Schrödinger equation is readily solved numerically for the case where $\Phi$ may be set to zero at the boundaries of the simulation [1], the open nature of the semiconductor device problem requires the formulation of nonzero boundary conditions to model the interaction of the device with particle reservoirs at the contacts, for both the time-dependent and the time-independent case [2]. The implementation of this type of boundary condition for the timedependent problem proves to be a formidable task.

*Address for correspondence: P.O. Box 345, Tainan, Taiwan 704, Republic of China. Fax: 886-6-2022377. 
In this paper, boundary conditions are imposed which model to second-order plane waves of constant amplitude incident at the contacts, and waves with modulated amplitude and phase exiting the contacts without reflection. This boundary condition scheme introduces irreversibility in the problem and thereby allows stable, steadstate solutions to be obtained, as well as transient solutions.

\section{FINITE-DIFFERENCE NUMERICAL METHOD FOR THE TIME-DEPENDENT SCHRÖDINGER EQUATION}

The evolution of a one-dimensional wave function $\Phi(x, t)$ is determined by the time-dependent Schrödinger equation

$$
\hat{H} \Phi(x, t)=\iota \hbar \frac{\partial}{\partial t} \Phi(x, t)
$$

the wave function $\Phi(x, t)$ can be given by

$$
\Phi_{j}^{n+1}=e^{-\iota \Delta t \hat{H} / \hbar} \Phi_{j}^{n}
$$

where $\Delta t, \mathrm{n}$, and $\mathrm{j}$ are the time interval, time index, and space index, respectively. Thus Eq. (2) can be approximated by

$$
\Phi_{j}^{n+1} \approx\left[1+\frac{-\iota \Delta t \hat{H} / \hbar}{1 !}+\frac{(-\iota \Delta t \hat{H} / \hbar)^{2}}{2 !}\right] \cdot \Phi_{j}^{n}
$$

with BenDaniel and Duke's effective Hamiltonian [3]

$$
\hat{H}=\frac{-\hbar^{2}}{2} \frac{\partial}{\partial x}\left[\frac{1}{m^{*}(x)} \frac{\partial}{\partial x}\right]+V(x)
$$

so as to preserve the continuity of the wave function.

The additional potential $|e| F x$ due to the external electric field is added directly to the potential profile of the structure. Equation (3) can be discredited with respect to time and space

$$
\begin{aligned}
\Phi_{j}^{n+1}= & \Phi_{j-2}^{n}\left[\frac{\Delta t^{2}}{2 \Delta x^{2}} \frac{-\hbar^{2}}{\Delta x^{2}} m_{a}(j-1) m_{b}(j-1)\right] \\
& +\Phi_{j-1}^{n}\left[\frac{\Delta t^{2}}{2 \Delta x^{2}} m_{a}(j-1) U_{j-1}+\left(-\iota \frac{\Delta t}{\hbar}-\frac{\Delta t^{2}}{2 \hbar^{2}} U_{j}\right) \frac{-\hbar^{2}}{\Delta x^{2}} m_{a}(j-1)\right]
\end{aligned}
$$




$$
\begin{aligned}
& +\Phi_{j}^{n}\left\{1+\left(-\imath \frac{\Delta t}{\hbar}-\frac{\Delta t^{2}}{2 \hbar^{2}} U_{j}\right) U_{j}+\frac{\Delta t^{2}}{2 \Delta x^{2}} \frac{-\hbar^{2}}{\Delta x^{2}}\left[m_{b}^{2}(j)+m_{a}^{2}(j)\right]\right\} \\
& +\Phi_{j+1}^{n}\left[\frac{\Delta t^{2}}{2 \Delta x^{2}} m_{b}(j+1) U_{j+1}+\left(-\iota \frac{\Delta t}{\hbar}-\frac{\Delta t^{2}}{2 \hbar^{2}} U_{j}\right) \frac{-\hbar^{2}}{\Delta x^{2}} m_{b}(j+1)\right] \\
& +\Phi_{j+2}^{n}\left[\frac{\Delta t^{2}}{2 \Delta x^{2}} \frac{-\hbar^{2}}{\Delta x^{2}} m_{b}(j+1) m_{a}(j+1)\right]
\end{aligned}
$$

where

$$
\begin{aligned}
m_{a}(j) & =\frac{1}{m_{j}^{*}+m_{j+1}^{*}} \\
m_{b}(j) & =\frac{1}{m_{j-1}^{*}+m_{j}^{*}} \\
U_{j} & =\frac{\hbar^{2}}{\Delta x^{2}}\left[m_{a}(j)+m_{b}(j)\right]+V_{j} .
\end{aligned}
$$

Equation (5) is the numerical solution of the time-dependent Schrödinger equation.

\section{BOUNDARY CONDITIONS}

\section{FOR THE TIME-DEPENDENT ANALYSIS}

In solving the Eq. (5), boundary conditions must be specified at the left and right simulation boundaries. It is desirable to set boundary conditions at the inflow point which correspond to incident plane waves of constant amplitude, to model the incident flux of carriers from particle reservoirs. However, at the inflow point there also exists a reflected wave whose characteristics are not known until the simulation develops. Therefore, the boundary condition should also minimize reflection of this wave at the boundary, to model particles leaving the simulation region. In this manner irreversibility is introduced, since the system loses memory of particles that have exited from the boundary, while the incident distribution is unaffected by these particles.

The approach taken here is to assume that at $t=0$ (time is redefined to reference zero at the beginning of each time step), the wave function at the boundary is given by (for the case of particles incident from the left)

$$
\Phi=A e^{\imath k x}+B(x) e^{-\imath k x}
$$


where $\Phi$ here represents only a single incident state, $\Phi_{i}$, in the ensemble. It is assumed that $\mathbf{A}$ is a constant to model the interaction with the reservoir, however, the envelop function of the reflected wave, $B(x)$, is allowed to vary with $x$, since, in general, the amplitude of the wave reflected from structure will vary with space and time. If the further assumption is made that to a good approximation the variation of $B(x)$ near the boundary may be regarded as a secondorder term, then the time-dependent Schrödinger equation at the boundary becomes

$$
\begin{aligned}
\iota \hbar \frac{\partial \Phi}{\partial t} & =\frac{\hbar^{2} k^{2}}{2 m^{*}} \Phi+\iota \frac{\hbar^{2} k}{m^{*}} \frac{\partial}{\partial X} B(X) e^{-\imath k X}+\frac{\hbar^{2}}{2 m^{*}} \frac{\partial^{2}}{\partial X^{2}} B(X) e^{-\imath k X} \\
& =\frac{\hbar^{2} k^{2}}{2 m^{*}} \Phi+r(X) e^{-\imath k X} \\
& =E \Phi+r(X) e^{-\imath k X}
\end{aligned}
$$

where

$$
r(X)=\iota \frac{\hbar^{2} k}{m^{*}} \frac{\partial}{\partial X} B(X)+\frac{\hbar^{2}}{2 m^{*}} \frac{\partial^{2}}{\partial X^{2}} B(X)
$$

where the potential energy at the boundary does not appear since $V$ is zero at the inflow boundary. The solution for $\Phi$ at the future time may be approximately written as

$$
\Phi(t=\Delta t) \approx \Phi(t=0) e^{(-\iota E / \hbar) \Delta t}+R(X) e^{-\imath k X} \Delta t
$$

In this equation, the first term on the right gives the time advancement expected for an energy eigenstate. The second term gives the modification of $\Phi$ due to the local variation of the reflected wave.

At the outflow point, only a transmitted wave exiting the boundary is expected, however the amplitude of this wave will, in general, not be constant. Therefore at the outflow, the wave function is assumed to be

$$
\Phi=C(x) e^{-\imath k_{0} x}
$$

where the wave vector $k_{o}$ differs from $k$ appearing in Eq. (7) as follows (again for the case of an incident wave from the left):

$$
k_{o}=\sqrt{2 m^{*}\left(E-V_{\max }\right)} / \hbar
$$


Again assuming that to a good approximation the variation of $\mathbf{C}(\mathbf{x})$ near the boundary may be regarded as a second-order term, then the time-dependent Schrödinger equation at the boundary becomes

$$
\begin{aligned}
\iota \hbar \frac{\partial \Phi}{\partial t} & =\frac{\hbar^{2} k_{o}^{2}}{2 m^{*}} \Phi-\iota \frac{\hbar^{2} k_{o}}{m^{*}} \frac{\partial}{\partial x} C(x) e^{-\imath k_{o} x}-\frac{\hbar^{2}}{2 m^{*}} \frac{\partial^{2}}{\partial x^{2}} C(x) e^{-\imath k_{o} x}+V(x) \Phi \\
& =\frac{\hbar^{2} k^{2}}{2 m^{*}} \Phi-t(x) e^{-\imath k_{o} x} \\
& =E \Phi-t(x) e^{-\imath k_{o} x}
\end{aligned}
$$

where

$$
t(x)=\iota \frac{\hbar^{2} k_{o}}{m^{*}} \frac{\partial}{\partial X} C(x)+\frac{\hbar^{2}}{2 m^{*}} \frac{\partial^{2}}{\partial x^{2}} C(x)
$$

The time-dependent Schrödinger equation may be approximately solved to give the update:

$$
\Phi(t=\Delta t) \approx \Phi(t=0) e^{(-\iota E / h) \Delta t}-T(x) e^{-\iota k_{0} x} \Delta t
$$

The boundary updates given by Eqs. (9) and (14) are used to set boundary conditions of Eq. (5). It is seen that in order to use these expressions, $R(x)$ and $T(x)$ must be determined.

We can obtain $\Phi_{4}^{n+1}$ and $\Phi_{3}^{n+1}$ from Eq. (5) and then $\Phi_{4}^{n+1}$ and $\Phi_{3}^{n+1}$ can be expressed by Eq. (9)

$$
\begin{aligned}
& \Phi_{4}^{n+1} \approx \Phi_{4}^{n} e^{(-\iota E / \hbar) \Delta t}+R\left(X_{4}\right) e^{-\iota k X_{4}} \Delta t \\
& \Phi_{3}^{n+1} \approx \Phi_{3}^{n} e^{(-\iota E / \hbar) \Delta t}+R\left(X_{3}\right) e^{-\iota k X_{3}} \Delta t
\end{aligned}
$$

where $R\left(x_{4}\right)$ and $R\left(x_{3}\right)$ can be show by

$$
\begin{aligned}
& R\left(X_{4}\right) \approx\left(\Phi_{4}^{n+1}-\Phi_{4}^{n} e^{(-\iota E / \hbar) \Delta t}\right) / \Delta t e^{-\imath k X_{4}} \\
& R\left(X_{3}\right) \approx\left(\Phi_{3}^{n+1}-\Phi_{3}^{n} e^{(-\iota E / \hbar) \Delta t}\right) / \Delta t e^{-\iota k X_{3}}
\end{aligned}
$$

When $R\left(x_{4}\right)$ and $R\left(x_{3}\right)$ are determined and then $R\left(x_{2}\right)$ and $R\left(x_{1}\right)$ can be determined by linear extrapolate method. Then $R\left(x_{2}\right)$ and $R\left(x_{1}\right)$ are known, that $\Phi_{2}^{n+1}$ and $\Phi_{1}^{n+1}$ can obtain by Eq. (9). A similar procedure is used to determine $T\left(x_{\max -1}\right)$ and $T\left(x_{\max }\right)$, except in this case it is not necessary to subtract out the incident wave component. 


\section{RESULTS}

A psudomorphic GaAs/AlGaAs double-barrier quantum well structure, the well width is $50 \AA$ and the effective mass used is
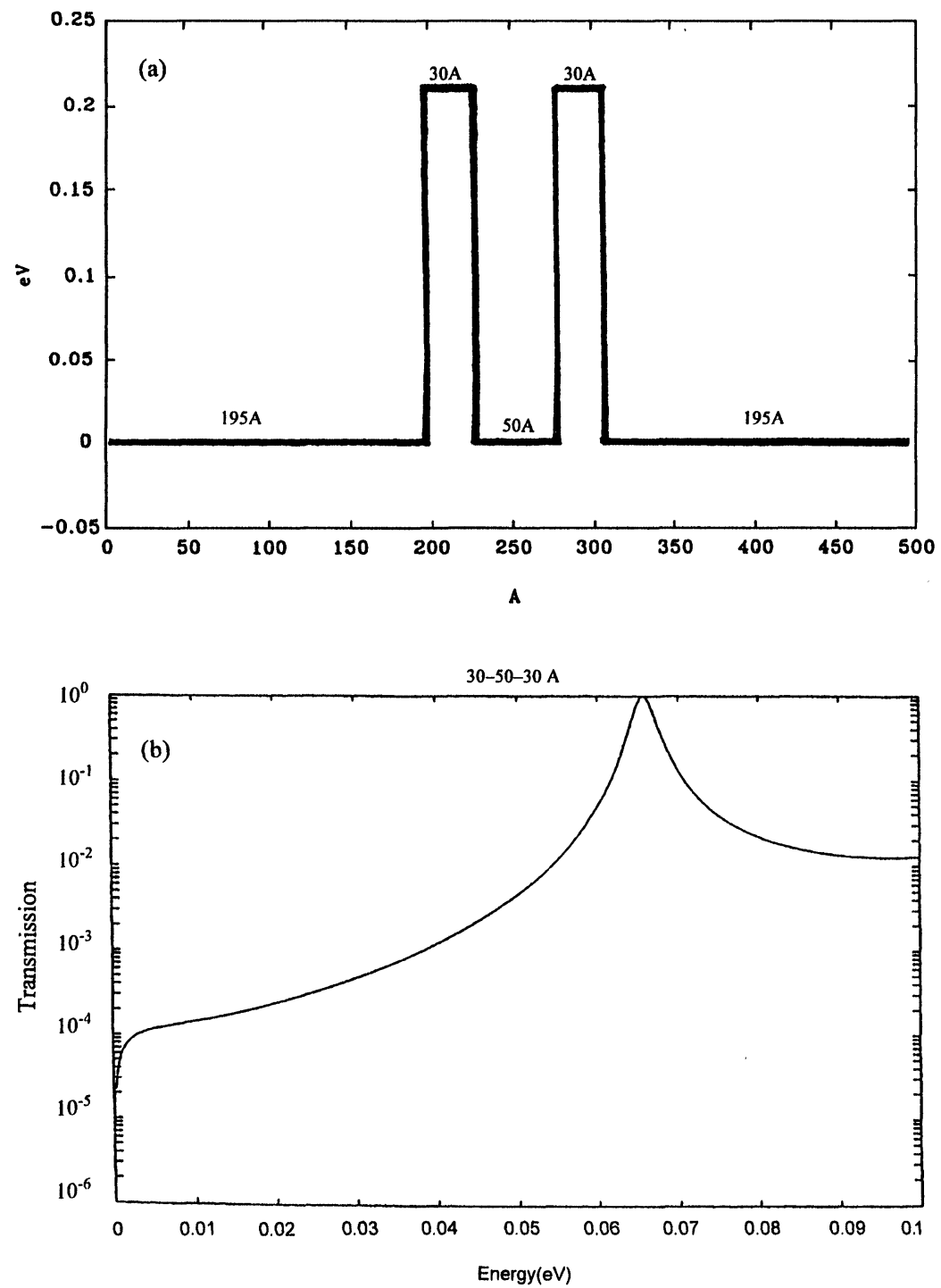

FIGURE 1 (a) potential profile (b) the transmission coefficient-electron energy curve at zero bias. 
$m^{*}=0.067 \mathrm{~m}_{\mathrm{o}}$, the barrier widths are $30 \AA$ and the effective mass used is $m^{*}=0.097 \mathrm{~m}_{0}$, the conduction-band discontinuity is assumed $0.212 \mathrm{eV}$. Figure 1(a) shows the potential profile of the structure. Figure 1(b) show the transmission coefficient-electron energy curve at

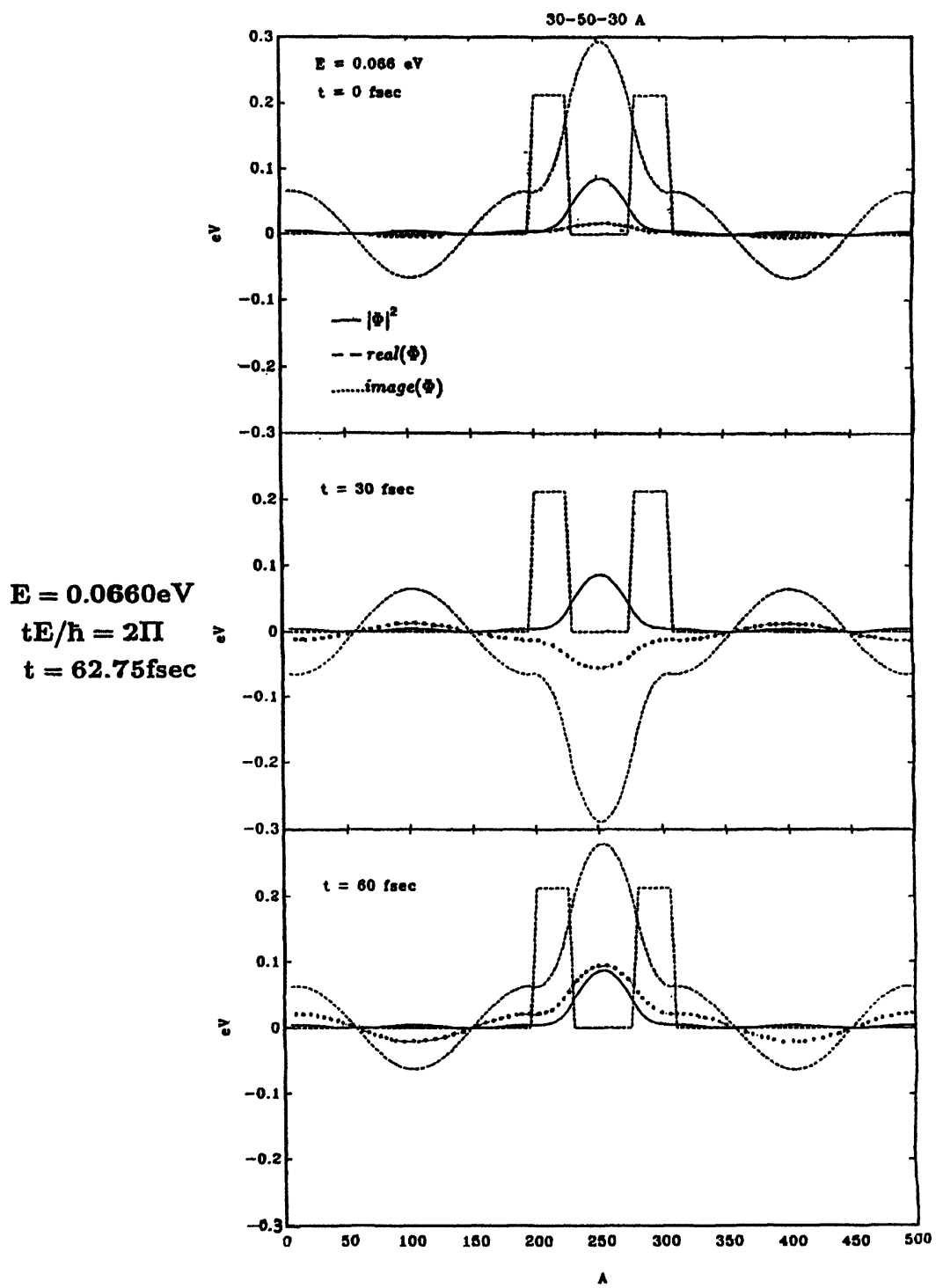

FIGURE 2 The $|\Phi|^{2}, \operatorname{real}(\Phi)$ and $\operatorname{image}(\Phi)$ of $\mathrm{E}=0.066 \mathrm{eV}$ varies of time at zero bias. 
zero bias. When the transmission coefficient has maximum, that the energy state is a quasi-bound state. Figures 2 and 3 show the $|\Phi|^{2}$, $\operatorname{real}(\Phi)$ and image $(\Phi)$ varies of the quasi-bound state $(\mathrm{E}=0.066 \mathrm{eV})$

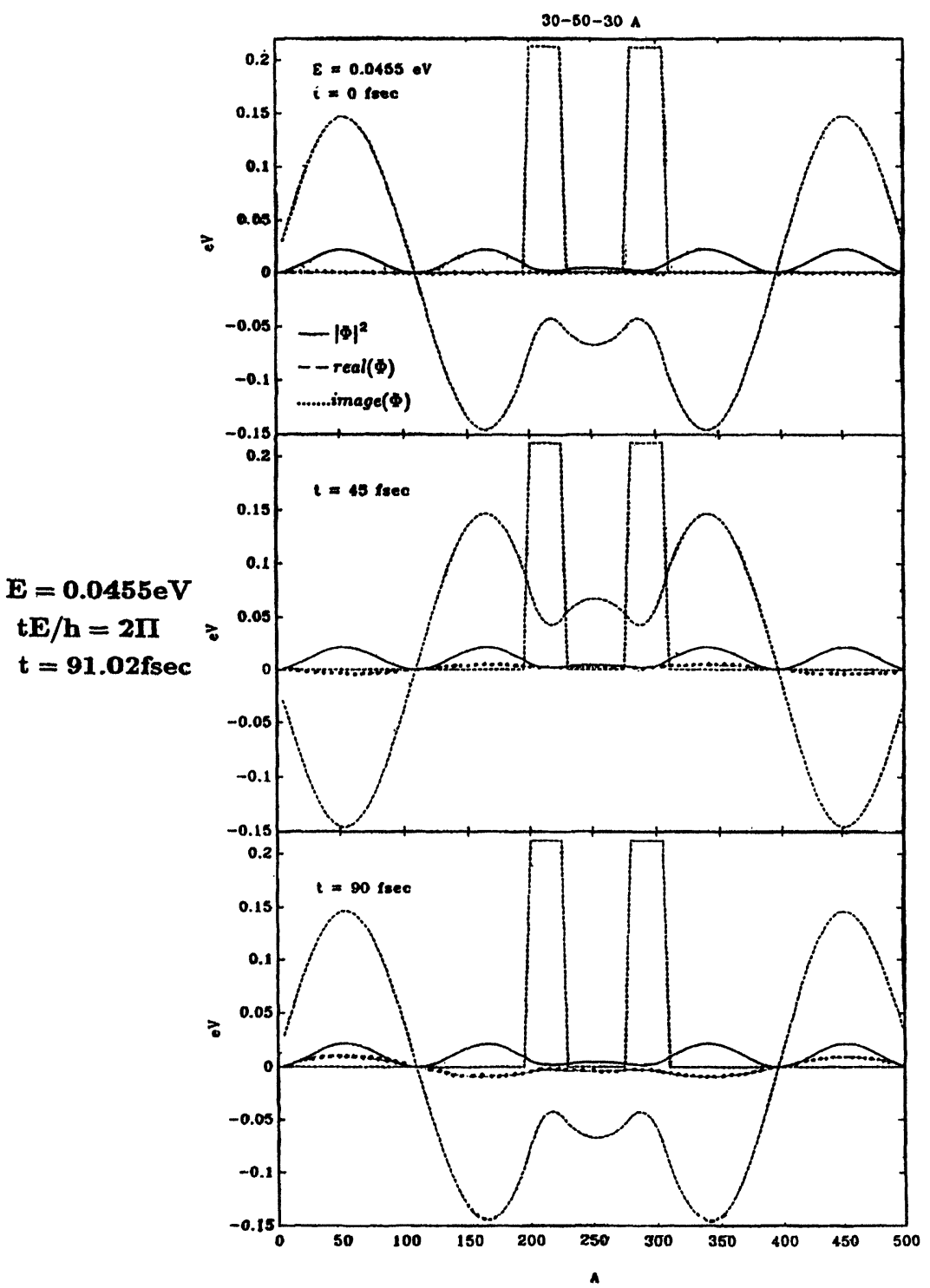

FIGURE 3 The $|\Phi|^{2}, \operatorname{real}(\Phi)$ and image $(\Phi)$ of $\mathrm{E}=0.0455 \mathrm{eV}$ varies of time at zero bias. 
$30-50-30 A$
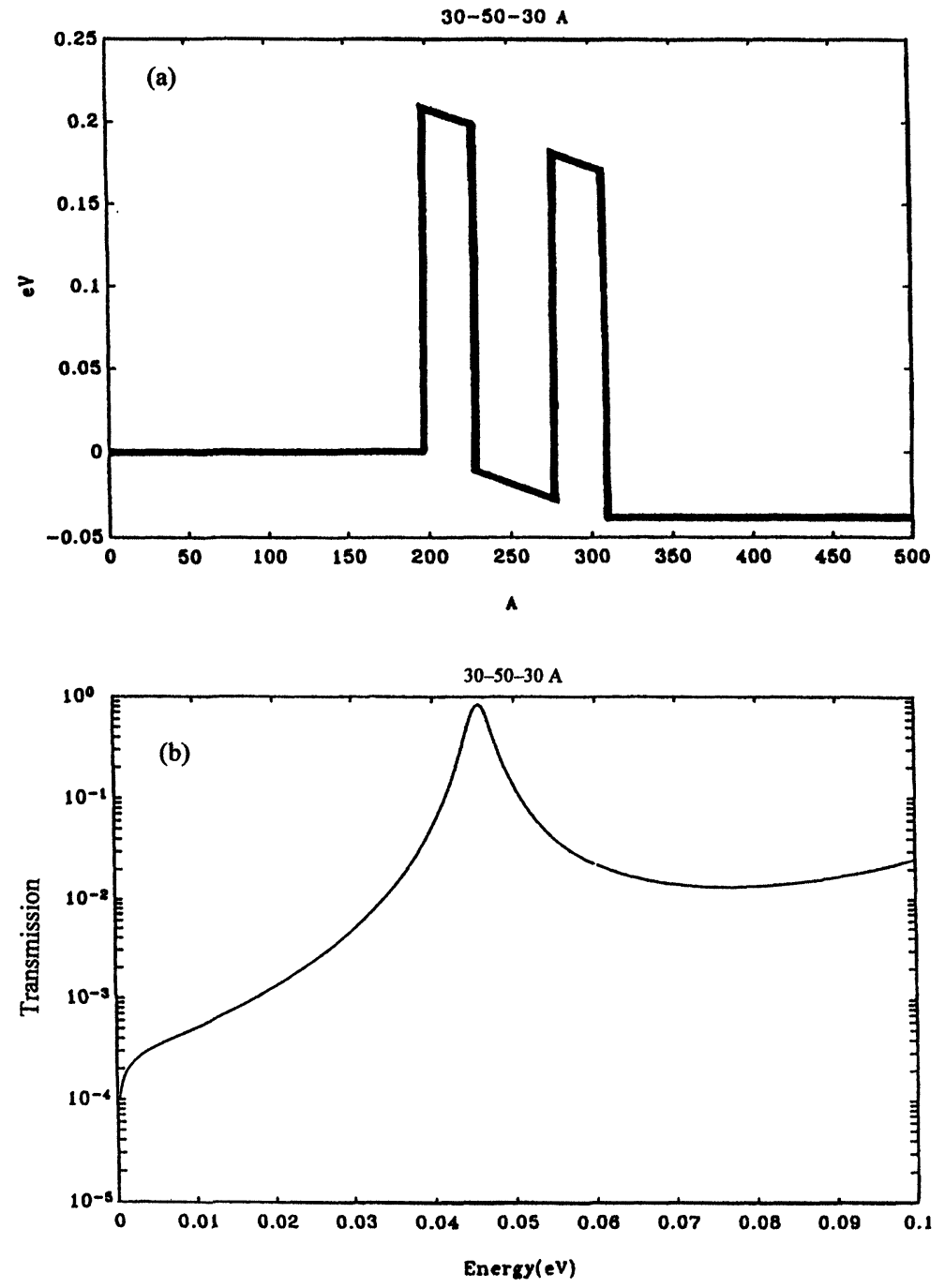

FIGURE 4 (a) potential profile (b) the transmission coefficient-electron energy curve at $-0.04 \mathrm{~V}$ bias.

and unquasi-bound state $(E=0.0455 \mathrm{eV})$, respectively. The $|\Phi|^{2}$ s of Figures 2 and 3 do not change in any time (steady solution) but the real $(\Phi)$ s and image $(\Phi)$ s change with cycles, when $\mathrm{E}=0.066 \mathrm{eV}$ the cycle approximate to $60 \mathrm{fsec}$ (numerical solution) and then $\mathrm{E}=0.0455 \mathrm{eV}$ the cycle approximate to $90 \mathrm{fsec}$ (numerical solution). The analytical 
solutions are $62.75 \mathrm{fsec}$ and $91.02 \mathrm{fsec}$ for $E=0.066 \mathrm{eV}$ and $E=$ $0.0455 \mathrm{eV}$, respectively.

Figure 4(a) shows the potential profile of the structure. Figure 4(b) show the transmission coefficient-electron energy curve at $-0.04 \mathrm{~V}$ bias, and then $\mathrm{E}=0.0455 \mathrm{eV}$ become quasi-bound state and $\mathrm{E}=0.066 \mathrm{eV}$ become unquasi-bound state. Figures 5 and 6 show the $|\Phi|^{2}$ of $E=0.066 \mathrm{eV}$ and $E=0.0455 \mathrm{eV}$ from zero bias to $-0.04 \mathrm{~V}$ bias, respectively. Figure 5 shows the wave function of $\mathrm{E}=0.066 \mathrm{eV}$ from quasi-bound state become unquasi-bound state and Figure 6 shows

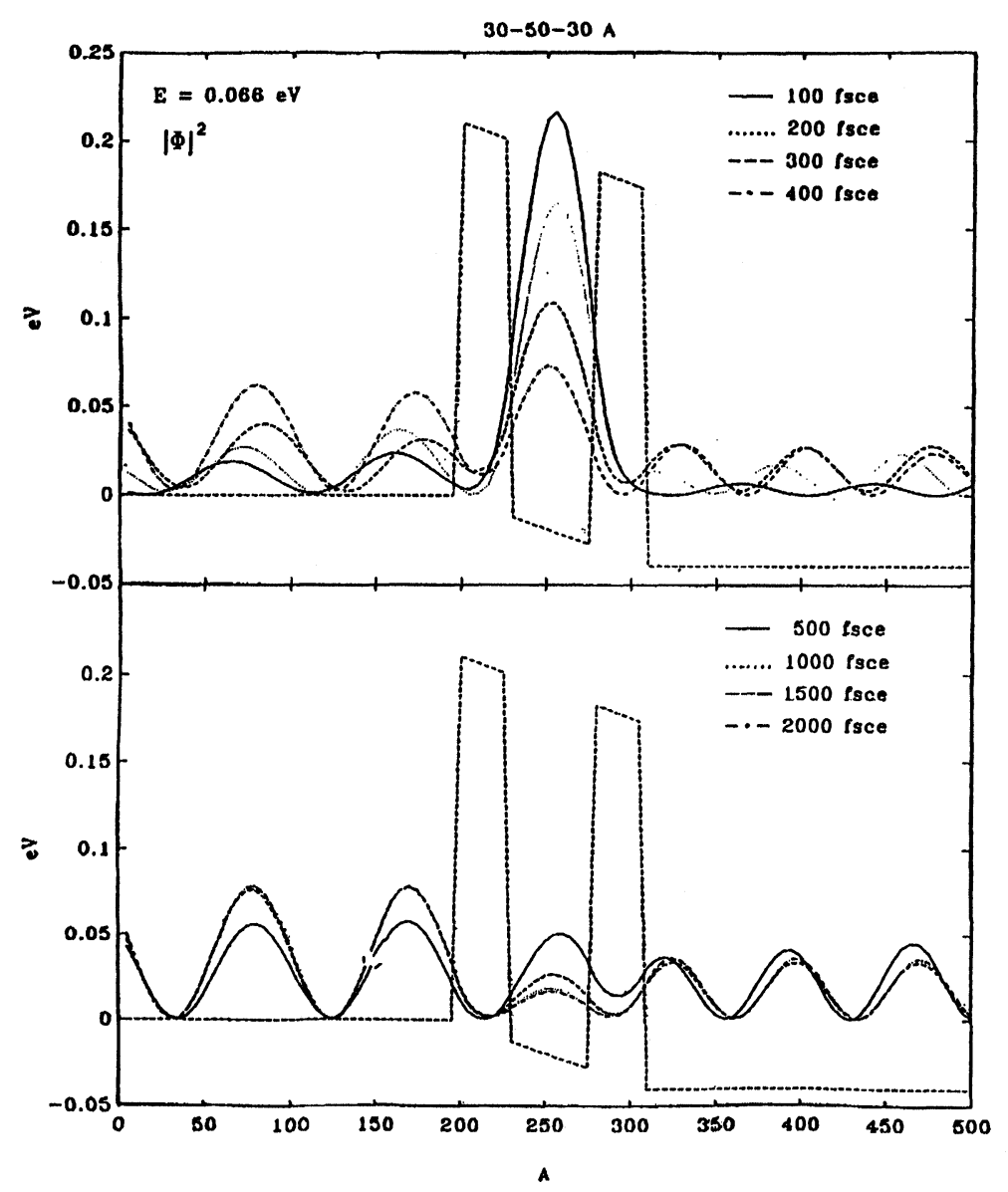

FIGURE 5 The $|\Phi|^{2}$ of $\mathrm{E}=0.066 \mathrm{eV}$ varies of time at $-0.04 \mathrm{~V}$ bias. 


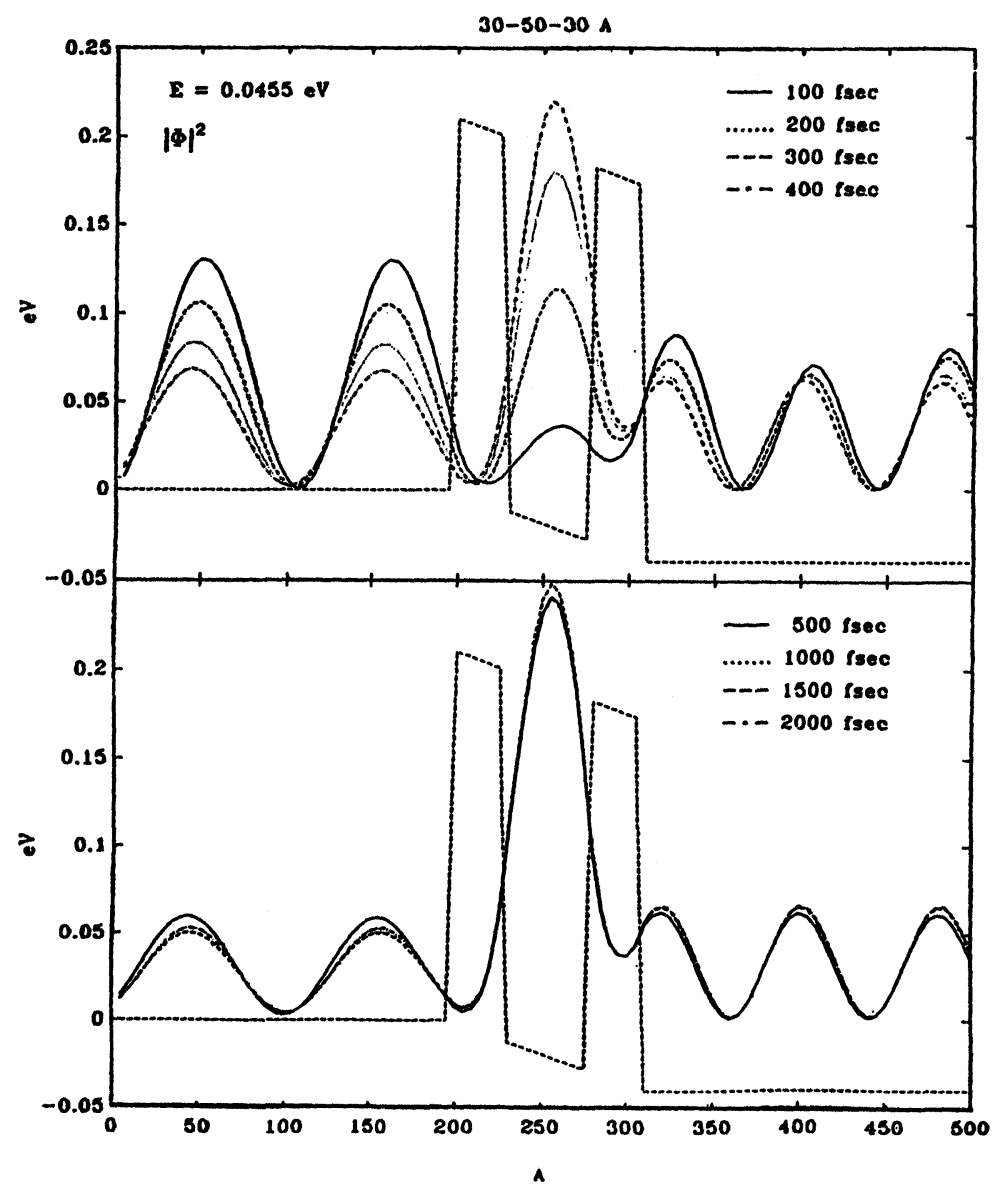

FIGURE 6 The $|\Phi|^{2}$ of $\mathrm{E}=0.0455 \mathrm{eV}$ varies of time at $-0.04 \mathrm{~V}$ bias.

the wave function of $\mathrm{E}=0.0455 \mathrm{eV}$ from unquasi-bound state become quasi-bound state. The changes of Figures 5 and 6 accord with the physical requirements.

\section{CONCLUSIONS}

A method of solving the time-dependent Schrödinger equation with boundary conditions modeling the interaction of the structure with 
particle reservoir at the contacts has been presented. This method is stable provided the time step is not too large.

Future work in this area should include an iterative formulation of the boundary condition scheme, which hopefully will allow larger time step to be take in the transient calculations and the I-V curve of the structure.

\section{References}

[1] Goldberg, A., Schey, H. M. and Schwartz, J. L. (1967). Am. J. Phys., 35, 177.

[2] Frensley, W. R. (1987). Phys. Rev., B36, 1570.

[3] BenDaniel, D. J. and Duke, C. B. (1966). Phys. Rev., 152, 683. 

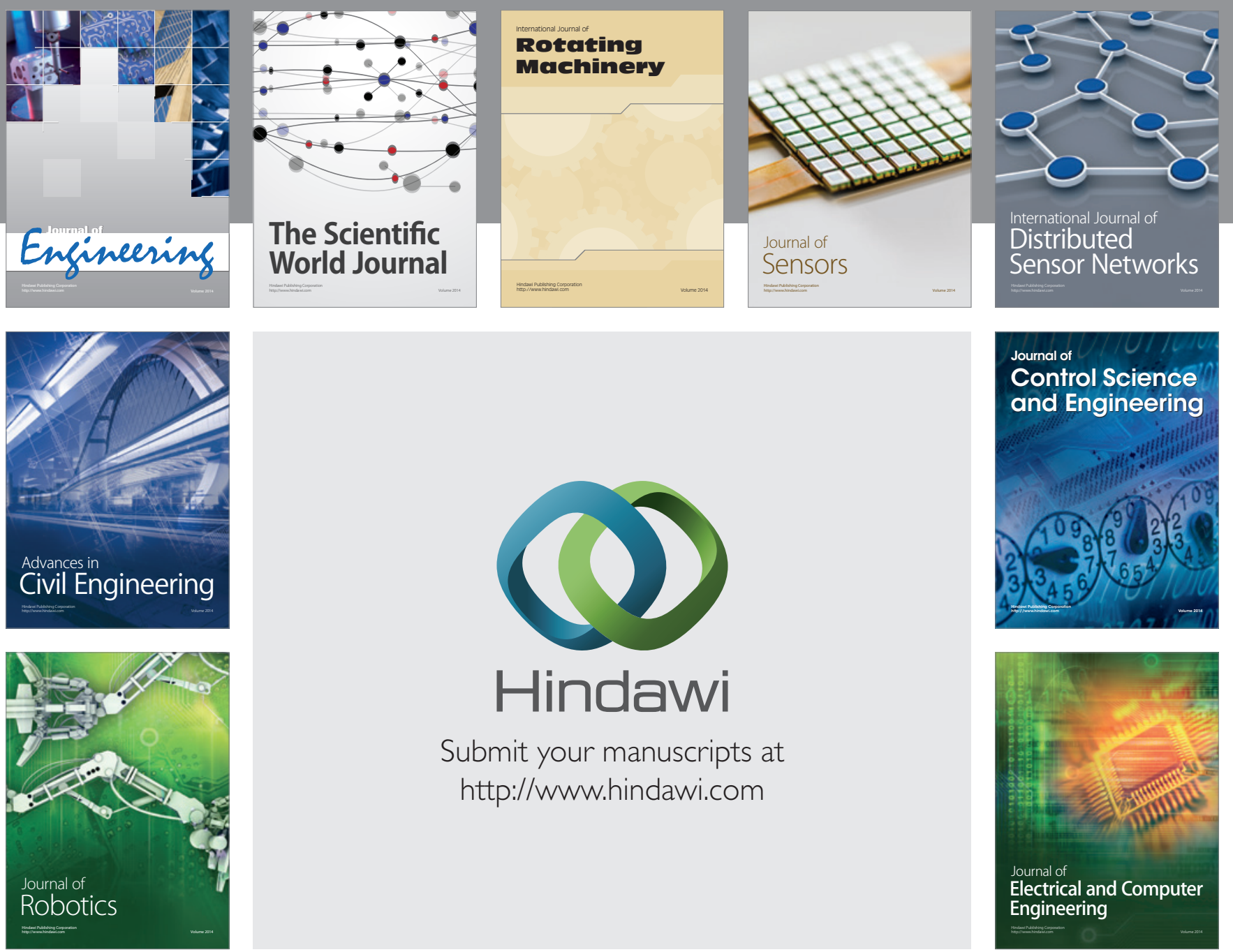

Submit your manuscripts at

http://www.hindawi.com
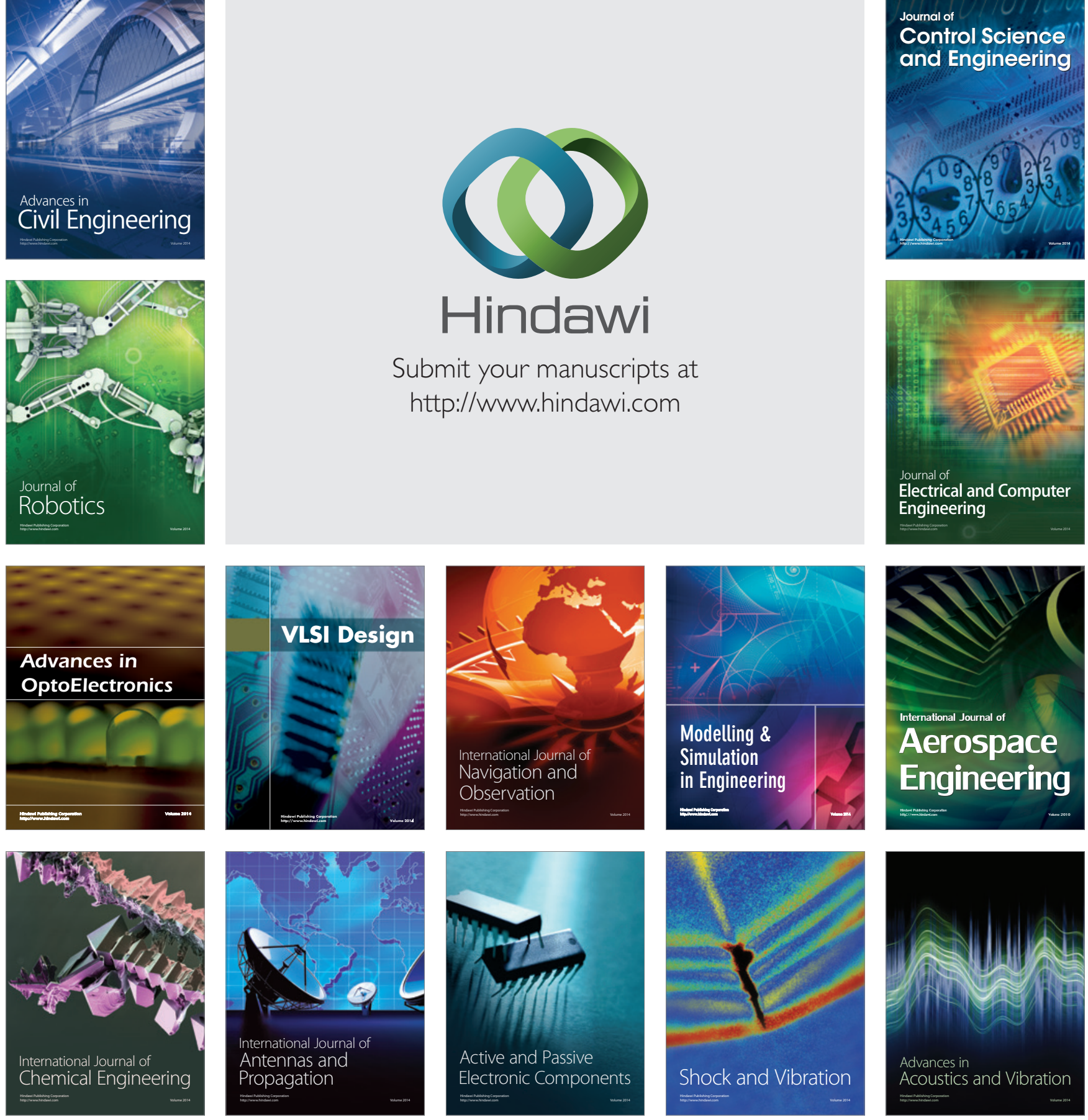